\title{
HERNIOPLASTIA COM TELA PRÉ-PERITONEAL NO TRATAMENTO DE HÉRNIA DE SPIEGEL: Relato de caso
}

\author{
Alfredo LIMA FILHO ${ }^{1}$ \\ Camila Carvalho CAVALCANTE ${ }^{2}$ \\ Diogo Ramalho Tavares MARINHO ${ }^{3}$ \\ Paulo Vinicius Alves LOPES ${ }^{4}$ \\ Rafael Calvão BARBUTO ${ }^{5}$ \\ Renan Farias Rolim VIANA ${ }^{6}$ \\ Ticiana de Magalhães Benevides LIMA $^{7}$
}

\author{
1 - Residente de Cirurgia Geral do Hospital das Clínicas da UFMG \\ (e-mail: alfredofilho@hotmail.com) \\ 2 - Residente de Cirurgia Geral do Hospital Luxemburgo \\ (email: camila_ccavalcante@hotmail.com) \\ 3 - Residente de Cirurgia Geral do Hospital das Clínicas da UFMG \\ (e-mail: drtm_7@hotmail.com) \\ 4 - Residente de Cirurgia Geral do Hospital das Clínicas da UFMG \\ (e-mail: paulovinicius.lopes@gmail.com) \\ 5 - Preceptor e Chefe da Residência de Cirurgia Geral do Hospital das Clínicas da UFMG \\ (email: rafaelbarbuto@gmail.com) \\ 6 - Residente de Cirurgia Geral do Hospital das Clínicas da UFMG \\ (e-mail:renanfrv@gmail.com) \\ 7 - Médica da Força Aérea Brasileira \\ (email: ticianabenevides@gmail.com)
}

Recebido em: 22/04/2015 - Aprovado em: 27/08/2015 - Disponibilizado em: 30/10/2015

RESUMO: As hérnias de Spiegel são um tipo incomum de hérnia, correspondendo a apenas $1 \%$ das hérnias abdominais. Decorrem da fraqueza congênita ou adquirida na parede abdominal, numa área denominada fáscia spigeliana, que é constituída pela camada aponeurótica limitada medialmente pelo reto abdominal e lateralmente pela linha arqueada. Representam um desafio diagnóstico, considerando-se que em geral se apresentam apenas com dor localizada e sem protrusão abdominal. O tratamento cirúrgico, seja aberto ou laparoscópico, se impõe diante do elevado risco de estrangulamento, devido ao estreito colo que em geral apresentam. Neste trabalho, relata-se a história de um paciente de 90 anos com dor em flanco esquerdo com dois anos de evolução e múltiplas consultas médicas, até então sem diagnóstico. A ultrassonografia revelou se tratar de hérnia de Spiegel. O paciente foi submetido a tratamento cirúrgico aberto, com correção do defeito herniário com tela de poliproprileno em posicionamento pré-peritoneal. Em acompanhamento ambulatorial, não se observaram sinais de recidiva. Apesar da tendência atual de priorizar o tratamento laparoscópico, ainda são poucos os estudos que comparam os seus benefícios em relação à abordagem convencional aberta.

Palavras-chave: Hernia. Spiegel. Hernioplastia.Tela. Pre-peritoneal.

ABSTRACT: Spigelian hernia is an uncommon kind of abdominal hernias and represents about $1 \%$ of the them. This variety of hernia occurs due a congenital or acquired weakness in the abdominal wall, in an area called spigelian fascia, that is composed of the aponeurotic layer between the rectus muscle medially and the linea semilunaris laterally. Spigelian hernia is a challenging diagnosis because generally the patients present just a localized abdominal pain without abdominal bulge. Surgical procedure is necessary, either open or laparoscopic, due the potential of incarceration because of its narrow neck. This paper describes the history of a 90 years old man with abdominal pain in the flank along two years, without diagnosis, even with several doctor visits. The ultrasonography confirmed the diagnostic of Spigelian hernia. The patient was underwent to open approach with a preperitoneal mesh of polypropylene over the aponeurotic defect. In the follow-up, there was no sign of recidive. Instead of the current trend to use laparoscopic surgery, there are few studies comparing the benefits of laparoscopic and conventional open surgery.

Key-words: Hernia. Spigelian, Hernioplasty. Mesh. Preperitoneal. 


\section{INTRODUÇÃO}

A hérnia de Spiegel é incomum e corresponde a aproximadamente $1 \%$ das hérnias da parede abdominal. Ocorre como resultado de fraqueza adquirida ou congenita na fáscia spigeliana, que é composta da camada de aponeurose delimitada medialmente pelo reto abdominal e lateralmente pela linha semilunar. Em geral, são pequenas e, quando adquiridas, se desenvolvem durante a quarta e sétima década de vida. (SABINSTON JR; TOWNSEND, 2011; SPANGEN, 1984)

Na maioria das vezes, ocorrem sobre ou abaixo da linha arqueada, onde já não existe camada posterior da fáscia do reto abdominal. Em geral, são do tipo intraparietal, e não apresentam, pois, protrusão palpável, tendo a dor localizada como único sintoma, o que dificulta o diagnóstico (SABINSTON JR; TOWNSEND, 2011; SPANGEN, 1984, 1989; STOPPA, 1997).

\section{DESCRIÇÃO DO CASO}

Paciente G.O.S., 90 anos, sexo masculino, foi encaminhado ao ambulatório Parede Abdominal e Retroperitônio do HCUFMG com queixa de massa abdominal dolorosa. O quadro já havia dois anos de evolução, com piora aos esforços.

Sua história prévia contemplava diabetes mellitus tipo II e hipertensão arterial sistêmica. Já seu passado cirúrgico continha hemorroidectomia, ressecção transuretral de próstata por hiperplasia prostática benigna e, por fim, hérnia inguinal à direita tratada com hernioplastia inguinal. $\mathrm{Na}$ ocasião, referia uso de Enalapril 20mg BID e Metformina 500mg BID. Sem tabagismo ou etilismo. Ao exame físico, apresentava abdome doloroso a palpação em flanco esquerdo, com abaulamento em borda lateral do musculo reto abdominal, que se pronunciava à manobra de Valsalva. Aventada a hipótese de hérnia de Spigel, que se confirmou através da ultrassonografia.

Após estudo do caso, o grupo de Parede e Retroperitônio decidiu pela intervenção cirúrgica por laparotomia mediana infraumbilical, objetivando redução do conteúdo herniário e correção do defeito aponeurótico com uso de tela de polipropileno posicionada no espaço pré-peritoneal.

Durante o ato cirúrgico, foi evidenciada presença saco herniário com tecido adiposo pré-peritoneal, contido pela aponeurose do oblíquo externo. O conteúdo herniário foi reduzido e o saco herniário ressecado. Em seguida, o defeito aponeurótico foi fechado com sutura de poliproprileno e a tela de polipropileno foi posicionada no espaço pré-peritoneal.

O paciente evoluiu bem e recebeu alta no terceiro dia pós-operatório assintomático. Em seu retorno, no $18^{\circ}$ dia pós-operatório, não havia sinais de recidiva ou infecção. 
FIGURA 01- Defeito na parede abdominal com conteúdo herniário já reduzido .

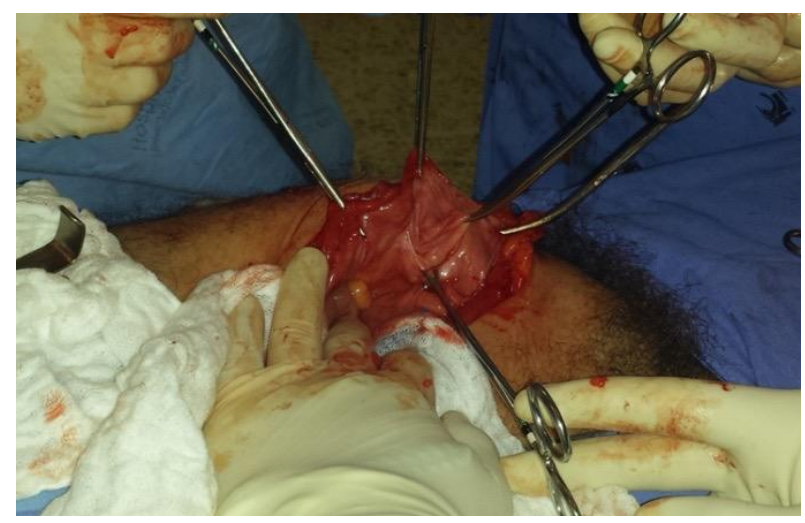

Fonte: Fotos tiradas no próprio serviço

FIGURA 2 - Visão do defeito após dissecção de espaço pré-peritoneal

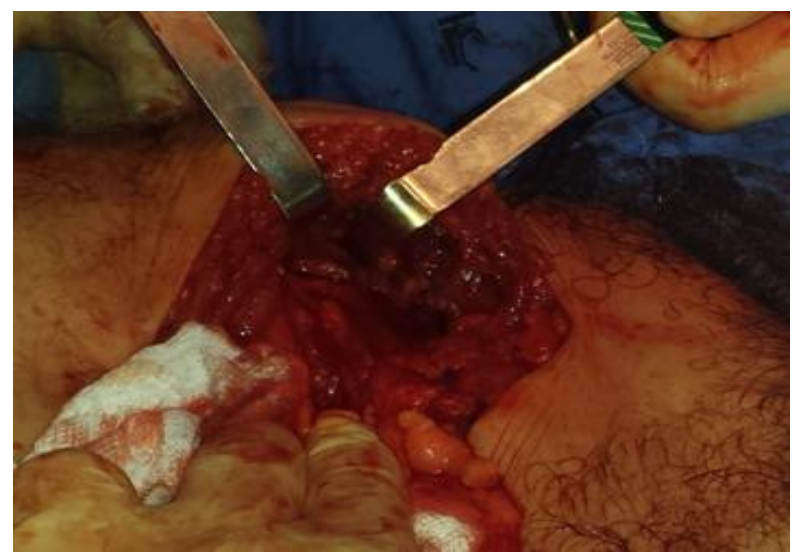

Fonte: Fotos tiradas no próprio serviço.

FIGURA 3 - Visão do defeito herniário tratado e tela de polipropileno posicionada.

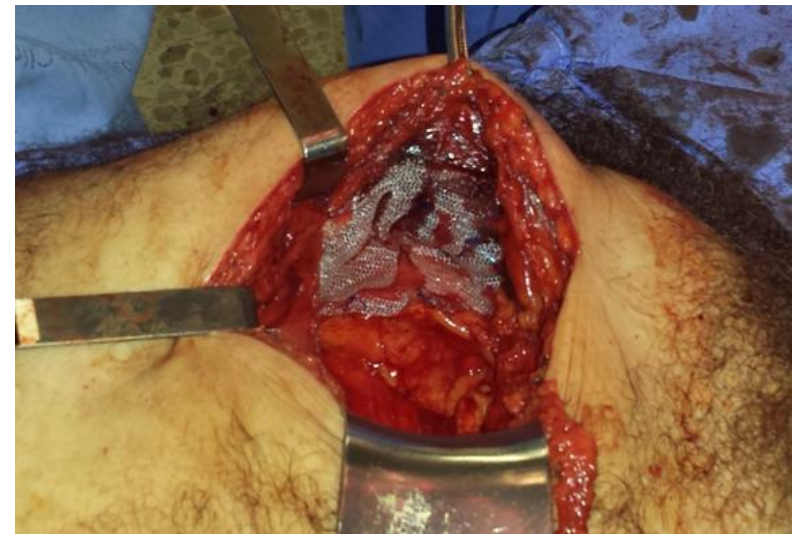

Fonte: Fotos tiradas no próprio serviço
FIGURA 4 -Ferida operatória no $18^{\circ}$ dia pósoperatorio.

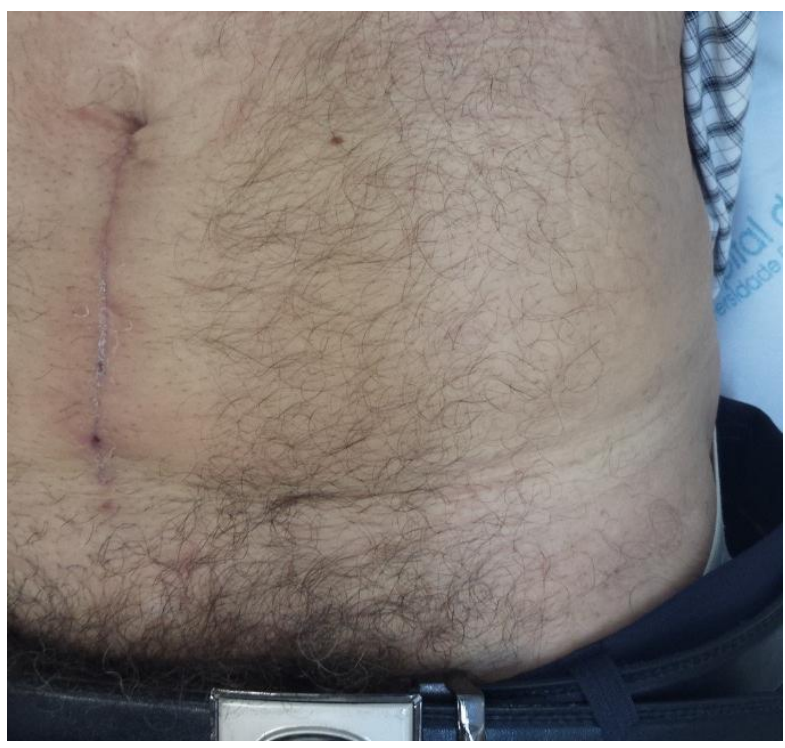

Fonte: Fotos tiradas no próprio serviço

\section{DISCUSSÃO}

$\mathrm{O}$ anatomista e cirurgião Adrian Van der Spiegel (1578-1625) realizou a descrição anatômica da linha semilunar. Em 1764, J.T. Klinkosch descreveu uma hérnia ventral lateral localizada especificamente na linha semilunar de Spiegel (MOLES MORENILLA et al, 2005).

A maioria se situa abaixo do músculo oblíquo externo, entre as diferentes camadas musculares da parede abdominal, devido a esse fato são comumente denominadas de hérnias ocultas, intersticiais ou intraparietais. Uma pequena porcentagem é superficial, onde o saco herniário atravessa a aponeurose do músculo oblíquo externo, tornando-se subcutâneo e sendo facilmente detectável durante o exame físico. A dor é o sintoma mais frequente, sendo seguido pela presença de massa referida pelo paciente, que nem 
sempre é palpável. Podem apresentar alterações no trânsito intestinal dependendo do conteúdo do saco herniário. Alguns casos são assintomáticos. A tomografia computadorizada e a ultrassonografia podem ajudar no diagnóstico, principalmente no que diz respeito à localização do defeito, o tamanho e o conteúdo do saco herniário, informações que são importantes para eleger a abordagem cirúrgica mais adequada. 亡̀ necessário um alto índice de suspeita e uma exploração meticulosa para alcançar um diagnóstico correto (RUIZ DE LA HERMOSA, 2010).

O tratamento da hérnia de Spiegel deve ser cirúrgico, tendo em vista o risco de encarceramento com o colo estreito que em geral apresentam (SKANDALAKIS et al, 1993; SABINSTON JR; TOWNSEND, 2011). O tratamento tradicional baseia-se em herniorrafia aberta anterior, usando aproximação direta dos músculos, telas e próteses (SKANDALAKIS, 2006; SPANGEN, 1989). Atualmente, a videolaparoscopia tem ganhado destaque já que também permite o uso de telas e impõe menor trauma cirúrgico ao paciente, favorecendo a recuperação pós-operatória. SKANDALAKIS et al, 2006; BAUCOM et al, 2009.). Neste trabalho, o paciente foi submetido a herniorrafia aberta com tela préperitoneal e ficou internado por três dias, tendo em vista, seu elevado risco associado às suas comorbidades.

\section{CONCLUSÃO}

O diagnóstico da hérnia de Spiegel é difícil em muitas ocasiões por ser do tipo intersticial. Há poucas publicações sobre esse assunto na literatura brasileira. $\mathrm{O}$ conhecimento desse tipo de hérnia interessa a várias especialidades médicas e se configura um desafio diagnóstico para o médico generalista, o radiologista e o cirurgião, que devem suspeitar de sua presença quando frente a dores abdominais atípicas. A ultrassonografia e a tomografia computadorizada são grandes ferramentas diagnósticas diante de casos em que somente o exame físico não pode descartar ou afirmar a presença da hérnia de Spiegel. Seu tratamento é eminentemente cirúrgico, destacando-se a modalidade de tratamento laparoscópica, já que proporciona menor trauma cirúrgico e, portanto, menor tempo de internação. Trabalhos adicionais são necessários para comparar a modalidade de tratamento tradicional aberta com a videolaparoscópica.

\section{REFERÊNCIAS}

BAUCOM, C.; NGUYEN, M. H.; SLAKEY, D.Minimally Invasive Spigelian Hernia

Repair. JSLS; v. 13, n. 2, p. 263-268, 2009.

CERDÁN, R.; BLÁZQUEZ, C.;

BARRANCO j.; Bernal Jaulín, J y Duque Mallén, M.; Hernia de Spiegel. Revisión a propósito de 8 casos. Rev Cubana Cir; v. 44, n.4, 2005. 
LARSON D.W; Farley DR. Spigelian

hernias: repair and outcome for 81

patients. World J Surg. 2002;26:1277-81.

MOLES MORENILLA, L. et al . Hernia de Spiegel en España: Análisis de 162 casos.Rev. esp. enferm. dig., Madrid, v. 97, n. 5, maio 2005 . Disponivel em: $<$ http://scielo.isciii.es/scielo.php?script=sci_ar ttext\&pid=S1130-

01082005000500006\&lng=es\&nrm=iso >

Acesso em:

20 abr. 2015. <http://dx.doi.org/10.4321/S1

130-01082005000500006>

RUIZ DE LA HERMOSA, A. et al . Hernias de Spiegel: Nuestra experiencia y revisión de la literatura. Rev. esp. enferm.

dig., Madrid, v. 102, n. 10, oct. 2010

- Disponível em

$<$ http://scielo.isciii.es/scielo.php?script=sci_ar

ttext\&pid=S1130-

01082010001000003\&lng=es\&nrm=iso>.

Acesso em 20 abr. 2015.

SABISTON JR., D. C., TOWNSEND, M. C.

Tratado de Cirurgia. 19. ${ }^{a}$ ed. Rio de

Janeiro: Guanabara Koogan, 2011.

SKANDALAKIS L.J; GADACZ T.R;

MANSBERGER, A.R; MITCHELL, W.E;

COLBORN, G.L; SKANDALAKIS,

J.E. Mondern hernia repair. The

embryological and anatomical basis of surgery. 2nd ed. New York: Parthenon;

1993. p 71-4.

SKANDALAKIS, P.N; ZORAS, O;

SKANDALAKIS, J,E; MIRILAS P.

Spigelian hernia: surgical anatomy, embryology, and technique of repair. Am

Surg. 2006;72:42-48.

SPANGEN L. Spigelian hernia. Surg Clin North Am. 1984;64:351-66.

SPANGEN L. Spigelian hérnia. World J Surg. 1989;13:573-80.

STOPPA R. Spigelian hernias. In: Chevrel JP, editor. Hernia ans surgery of the abdominal wall. Berlin: Springer; 1997. p.267-9. 